\title{
Multiscale simulations of primary atomization
}

\author{
Gaurav Tomar ${ }^{\mathrm{a}, \mathrm{b}}$, Daniel Fuster ${ }^{\mathrm{a}, \mathrm{b}}$, Stéphane Zaleski ${ }^{\mathrm{a}, \mathrm{b}, *}$ and \\ Stéphane Popinet ${ }^{\mathrm{c}}$ \\ ${ }^{a}$ UPMC Univ Paris 06, UMR 7190, Institut Jean Le Rond d'Alembert, F-75005 \\ Paris, France. \\ ${ }^{\mathrm{b}}$ CNRS, UMR 7190, Institut Jean Le Rond d'Alembert, F-75005 Paris, France. \\ ${ }^{\mathrm{c}}$ National Institute for Water and Atmospheric research, P.O. Box 14-901, \\ Kilbirnie, Wellington, New Zealand.
}

\begin{abstract}
A liquid jet upon atomization breaks up into small droplets that are orders of magnitude smaller than its diameter. Direct numerical simulations of atomization are exceedingly expensive computationally. Thus, the need to perform multiscale simulations. In the present study, we performed multiscale simulations of primary atomization using a Volume-of-Fluid (VOF) algorithm coupled with a two-way coupling Lagrangian particle-tracking model to simulate the motion and influence of the smallest droplets. Collisions between two particles are efficiently predicted using a spatial-hashing algorithm. The code is validated by comparing the numerical simulations for the motion of particles in several vortical structures with analytical solutions. We present simulations of the atomization of a liquid jet into droplets which are modeled as particles when away from the primary jet. We also present the probability density function of the droplets thus obtained and show the evolution of the PDF in space.
\end{abstract}

Key words: Atomization, Volume of fluid, Lagrangian Particle Tracking, MultiScale Simulations.

\section{Introduction}

A large set of physical systems of industrial and scientific relevance involve interactions between phenomena occurring at various scales. Disparate scales are seen in situations like micro and nano-fluidics, particle-laden flows, combustion

* Email: stephane.zaleski@upmc.fr, Tel: +33 1 44272558, Fax: +33 144275259. 
chambers, spray drying and atmospheric flows $[1,2]$. Numerical simulations of a physical process involving temporal and spatial scales separated by orders of magnitude require huge computational resources and time. For example, at nano-scales molecular forces are dominant and govern the flow physics, whereas at micro-scales viscous forces are dominant and at even larger macroscales inertial forces also need to be incorporated. Therefore, hybrid models are needed to resolve the physics (approximately or thoroughly) at different scales. Such hybrid multiscale methods involve merging of models valid at various scales and interaction between them via an interface sharing information required for model closures.

Of the various models that bridge the different scales, perhaps the most challenging is the building of the interface between simulations at the nano/mesoscale to the macro-scale mechanics. The gross response at the small scales should be fused into the macro-scale. Various multiscale bridging methods have been developed [3], namely, the heterogeneous multiscale method [4], the variational multiscale method [5], the discontinuous Galerkin method [6] and the equation-free method [7]. Son and Dhir [8] performed numerical simulations of nucleate boiling by modeling the micro-film underneath the bubble using thinfilm equations incorporating the long-range van der Waals forces and phasechange. The micro-layer in nucleate boiling is responsible for a predominant fraction of heat transfer and phase change and also governs the macroscopic contact angle.

Multiscale simulations involving mesoscopic particles suspended in a macroscopic fluid have been performed using Eulerian-Eulerian and Eulerian-Lagrangian models $[9,10]$. In the Eulerian-Eulerian approach, the dispersed (small-scale) phase is collectively modelled using a concentration field. Statistical models are employed to simulate the cumulative effect of the dispersed phase on the suspension fluid. The concentration field is advected with the flow using a Hamilton-Jacobi type equation. In contrast, Eulerian-Lagrangian methods involve solving the continuous phase using numerical schemes such as Finite Differences, Finite Elements or Finite Volumes and performing discrete Lagrangian advection of the dispersed phase. The hydrodynamic forces acting on the particles are incorporated using empirical correlations and theoretical models. The effect of the particles on the underlying fluid is modeled as a source term in the momentum equations. A very low concentration of particles may not alter the flow field significantly but moderate concentrations modify flow structures. Ferrante and Elghobashi [11] studied the effect of micro-bubbles on vortical structures. Small bubbles are easily entrapped and convected in vortical structures. A moderate accumulation of bubbles was shown [11] to enhance the decay rate of the vorticity at the center of the vortex. Van Sint Annaland et al. [12] performed numerical simulations of gas-liquid-solid flows by combining a Front-Tracking algorithm for two-phase flows with a discrete particle method to model the solid dispersed phase. They 
demonstrated the effect of bubble-induced particle mixing and modification of the drag on the bubble during its ascent. In turbulent flows, particles are swept away by the swirling eddies and vortices. Particles alter flow dynamics leading to attenuation or enhancement of turbulence based on a non-dimensional particle momentum number $[13,14]$. The effect of particles on turbulence in the flow field has also been studied in [15]. Climent and Magnaudet [16] studied the effect of bubble dispersion on a mixing layer. Simulations were performed using a two-way coupling Eulerian-Lagrangian method. In two-way coupled Eulerian-Lagrangian methods, the effect of the motion of bubbles on the fluid momentum is incorporated by infusing a momentum source term in the Navier-Stokes equation for the fluid flow. Bubbles are initially captured in the vortices in the mixing layer and later dispersed upon an increase in their concentration. Bubbles enhance the destabilization process in the mixing layer and shorten the time required for the roll-up phenomenon.

A liquid jet breaks up into small droplets because of an interplay of several mechanisms active in the atomization process. The droplets broken-off from the liquid jet are orders of magnitude smaller than its diameter. With the advent of various sharp-interface-tracking algorithms [17-23], numerical simulations of the atomization processes have become possible [24-26]. Threedimensional temporal simulations of the breakup of a liquid jet by a coaxial high-speed gas have been reported in [24, 27] using the Volume-of-Fluid (VOF) method and by Tauber et al [25] using the Front-Tracking method. Both studies showed the formation of thin ligaments which subsequently break into droplets. Small droplets formed during the atomization process require high grid resolution thus increasing the computational cost several folds. The computational cost can be reduced by artificially removing the smallest droplets from regions of the computational domain far from the liquid jet. An unaccounted removal of the droplets formed makes the measurements of probability density function (PDF) of droplets impossible and may also lead to a loss of physics. Thus, a multiscale model is required to incorporate the essential effects of the droplets without investing enormous computational effort in completely resolving all the involved scales using interface tracking and a Navier-Stokes solver. Recently, Kim et al [26] performed multiscale simulations of the primary breakup of a liquid jet by a coaxial flow of gas. A variant of the Level-Set method was employed to solve the two-phase flow coupled with a Lagrangian spray model to track the droplets broken-off from the liquid jet.

We present here multiscale simulations of primary jet breakup using an EulerianLagrangian two-way coupling method. The discrete particle method has been implemented in Gerris [28, 29], a two-phase VOF solver with balanced force surface tension model and quad/octree adaptive mesh refinement. The momentum source term in the Navier-Stokes equation arising from the particles is smoothed using a Gaussian distribution function. Collisions between particles are efficiently predicted by implementing a spatial-hashing algorithm to 
identify particles in the vicinity for collision. The algorithm has been validated against various test cases. Finally, we present simulations of breakup of a liquid jet converting droplets into particles upon formation and back into VOF resolved droplets based on their proximity to the VOF interface. We present a PDF of the droplet sizes during the atomization process.

\section{Formulation}

\section{A. Governing Equations}

The Navier-Stokes equations for a two-phase incompressible flow modified to implicitly incorporate the boundary conditions at the interface can be written as,

$$
\rho\left[\partial_{t} \boldsymbol{u}+(\boldsymbol{u} \cdot \boldsymbol{\nabla}) \boldsymbol{u}\right]=-\boldsymbol{\nabla} p+\boldsymbol{\nabla} \cdot(2 \mu \boldsymbol{D})+\sigma \kappa \delta_{s} \boldsymbol{n}+\boldsymbol{\Phi}_{p},
$$

where $\boldsymbol{u}=(u, v, w)$ is the fluid velocity, $\rho \equiv \rho(\boldsymbol{x}, t)$ is the fluid density, $\mu \equiv \mu(\boldsymbol{x}, t)$ is the dynamic viscosity and $\boldsymbol{D}$, the deformation tensor, is defined as $D_{\mathrm{ij}} \equiv\left(\partial_{i} u_{j}+\partial_{j} u_{i}\right) / 2$. The surface tension force is non-zero only at the interface as signified by the Dirac delta function, $\delta_{s}$, with $\sigma, \boldsymbol{n}$ and $\kappa$ representing the surface-tension coefficient, the unit normal and the curvature at the interface, respectively. The momentum source term $\boldsymbol{\Phi}_{\boldsymbol{p}}$ represents the effect of the dispersed phase simulated using a Lagrangian approach.

The advection equation for density and the incompressibility condition are given by,

$$
\begin{gathered}
\partial_{t} \rho+\nabla \cdot(\rho \boldsymbol{u})=0, \\
\nabla \cdot \boldsymbol{u}=0 .
\end{gathered}
$$

The density and the viscosity field are obtained as

$$
\begin{aligned}
& \rho(c) \equiv c \rho_{1}+(1-c) \rho_{2} \text { and } \\
& \mu(c) \equiv c \mu_{1}+(1-c) \mu_{2}
\end{aligned}
$$

respectively where $c(\boldsymbol{x}, t)$ is the volume fraction. Here, $\rho_{1}, \rho_{2}$ and $\mu_{1}, \mu_{2}$ are the densities and viscosities of the first and second fluid, respectively. The volume fraction takes values between zero and one. The interface motion can be written in terms of the volume fraction as,

$$
\partial_{t} c+\nabla \cdot(c \boldsymbol{u})=0 .
$$


A two-way coupled Eulerian-Lagrangian approach for the dispersed phase comprises of computing the external and fluid forces on the particles and incorporating the effect of the particles as a source term in the Navier-Stokes equation $\left(\Phi_{p}\right)$. The governing equations of the motion of the particles are given by,

$$
\begin{gathered}
\frac{d \boldsymbol{x}^{i}}{d t}=\boldsymbol{v}^{i} \\
m_{p}^{i} \frac{d \boldsymbol{v}^{i}}{d t}=F_{D}+F_{I}+F_{A}+F_{L}+F_{e x t}
\end{gathered}
$$

where, $m_{p}^{i}\left(=\rho_{p}^{i} V^{i}\right), \boldsymbol{x}^{i}$ and $\boldsymbol{v}^{i}$ are the mass, position and the velocity of the $i$-th particle respectively. The density and volume of the particle are denoted by $\rho_{p}^{i}$ and $V^{i}$, respectively. The various forces $(F)[15,30]$ acting on the particle are summarized below:

(1) Drag force:

$$
F_{D}=-\frac{3}{4 d_{i}} C_{D}\left(R e_{p}^{i}\right) \rho V^{i}\left|\boldsymbol{v}^{i}-\boldsymbol{u}\right|\left(\boldsymbol{v}^{i}-\boldsymbol{u}\right),
$$

where $C_{D}$ is the drag coefficient as a function of the Reynolds number based on particle diameter, $R e_{p}^{i}=d_{i}\left|\boldsymbol{v}^{i}-\boldsymbol{u}\right| / \nu$. Here, $d_{i}$ is the diameter of the particle. The exact form of $C_{D}$ depends upon various other factors, for example, shape and surfactant concentration of the surface [30]. The form of $C_{D}$ used in the present study is prescribed later. The density and the dynamic viscosity of the carrier fluid are given by $\rho$ and $\nu$, respectively.

(2) Inertial force:

$$
F_{I}=\rho V^{i}\left[\frac{\partial \boldsymbol{u}}{\partial t}+(\boldsymbol{u} \cdot \nabla) \boldsymbol{u}\right]
$$

(3) Added mass force:

$$
F_{A}=\rho V^{i} C_{M}\left[\frac{\partial \boldsymbol{u}}{\partial t}+(\boldsymbol{u} \cdot \nabla) \boldsymbol{u}-\frac{d \boldsymbol{v}^{i}}{d t}\right]
$$

where $C_{M}$ is the added-mass coefficient. The added-mass coefficient, $C_{M}=$ $1 / 2$, is independent of the Reynolds number and the strength of the acceleration $[31,32]$.

(4) Lift force:

$$
F_{L}=-\rho C_{L} V^{i}\left(\boldsymbol{v}^{i}-\boldsymbol{u}\right) \times \boldsymbol{\omega} .
$$

Here, $\boldsymbol{\omega}=\boldsymbol{\nabla} \times \boldsymbol{u}$ is the vorticity and $C_{L}$ is the lift coefficient. The lift coefficient is a weakly increasing function of the Reynolds number and tends quickly towards the value one-half $[30,33]$. In all the simulations presented here, we use $C_{L}=1 / 2$.

(5) External forces may comprise the conservative and the non-conservative forces acting on the particle due to an externally applied field, namely, 
gravitational, electric or magnetic. For instance, the buoyancy force acting on the particle is given by,

$$
F_{\text {ext }}=\left(\rho_{p}-\rho\right) \boldsymbol{g}
$$

where $\boldsymbol{g}$ is the gravitational acceleration.

The momentum source term $\boldsymbol{\Phi}_{p}$ in the Eq.1 is given by,

$$
\boldsymbol{\Phi}_{p}=\lim _{V_{f} \rightarrow 0} \sum_{i=1}^{N_{p}} \frac{V^{i}}{V_{f}}\left[\rho_{p}^{i}\left(\boldsymbol{g}-\frac{d \boldsymbol{v}^{i}}{d t}\right)+\rho\left(\frac{D \boldsymbol{u}}{D t}-\boldsymbol{g}\right)\right],
$$

where $V_{f}$ is the control volume of the fluid containing $N_{p}$ particles [16].

\section{Numerical Modeling}

\section{A. Brief overview of the VOF algorithm}

The methodology adopted for solving the two-phase, sharp-interface, incompressible flow equations is presented in detail in [28, 29, 34]. We describe here briefly the algorithm implemented. A second-order accurate staggered time discretisation has been employed for the volume-fraction/density and pressure fields. Using the classical time-splitting projection method the discretized equations can be written as:

$$
\begin{aligned}
& \rho_{n+\frac{1}{2}}\left[\frac{\boldsymbol{u}_{\star}-\boldsymbol{u}_{n}}{\Delta t}+\boldsymbol{u}_{n+\frac{1}{2}} \cdot \nabla \boldsymbol{u}_{n+\frac{1}{2}}\right]=\boldsymbol{\nabla} \cdot\left[\mu_{n+\frac{1}{2}}\left(\boldsymbol{D}_{n}+\boldsymbol{D}_{\star}\right)\right] \\
& +\left(\sigma \kappa \delta_{s} \boldsymbol{n}\right)_{n+\frac{1}{2}} \\
& \frac{c_{n+\frac{1}{2}}-c_{n-\frac{1}{2}}}{\Delta t}+\nabla \cdot\left(c_{n} \boldsymbol{u}_{n}\right)=0 \\
& \boldsymbol{u}_{n+1}=\boldsymbol{u}_{\star}-\frac{\Delta t}{\rho_{n+\frac{1}{2}}} \nabla p_{n+\frac{1}{2}}, \\
& \boldsymbol{\nabla} \cdot \boldsymbol{u}_{n+1}=0 \text {. }
\end{aligned}
$$

Using equations (17) and (18), the Poisson equation governing the pressure field can be written as,

$$
\nabla \cdot\left(\frac{\Delta t}{\rho_{n+\frac{1}{2}}} \nabla p_{n+\frac{1}{2}}\right)=\nabla \cdot \boldsymbol{u}_{\star} .
$$


The Poisson equation (19) for the pressure is solved efficiently using a quad/octreebased multigrid iterative solver [28]. The discretized momentum-conservation equation (Eq. 15) is an Helmholtz-type equation and is solved using a variant of the multilevel Poisson solver employed to solve the pressure equation. The Crank-Nicholson discretization of the viscous terms described above is secondorder accurate in time and is unconditionally stable. The convective terms $\left(\boldsymbol{u}_{n+\frac{1}{2}} \cdot \boldsymbol{\nabla} \boldsymbol{u}_{n+\frac{1}{2}}\right)$ are computed using the Bell-Colella-Glaz [28, 35] secondorder unsplit upwind scheme which is stable for CFL numbers smaller than one.

The velocity, pressure and tracers are all collocated at the center of the computational finite-volume cell. The velocity and pressure fields are decoupled using an approximate projection method for the spatial discretization of the pressure correction equation and the associated divergence of the auxiliary velocity $[28,36]$. The time integration algorithm implemented in Gerris can be broadly divided into the following steps:

(1) The auxiliary cell-centered velocity field, $\boldsymbol{u}_{\star}$, is computed using equation (15).

(2) Pressure correction: An auxiliary face-centered velocity is obtained by averaging the cell-centered values on all the cell faces. The divergence of the velocity field is computed using the face-centered velocity for each control volume as the finite-volume approximation:

$$
\boldsymbol{\nabla} \cdot \boldsymbol{u}_{\star}=\frac{1}{\Delta} \sum_{f} \boldsymbol{u}_{\star}^{f} \cdot \boldsymbol{n}^{f},
$$

where, $\boldsymbol{n}^{f}$ is the unit normal vector to the face and $\Delta$ is the length scale of the control volume. The Poisson equation (19) is solved using the abovedefined velocity divergence to obtain the pressure field corresponding to a divergence-free velocity. Corrected face-velocities are computed using the pressure field as follows:

$$
\boldsymbol{u}_{n+1}^{f}=\boldsymbol{u}_{\star}^{f}-\frac{\Delta t}{\rho\left(c_{n+\frac{1}{2}}^{f}\right)} \nabla^{f} p_{n+\frac{1}{2}},
$$

where $c_{n+1 / 2}^{f}$ is obtained by averaging from the cell-centered values $c_{n+1 / 2}^{c}$ and $\nabla^{f}$ is a simple face-centered gradient operator (consistent at coarse/fine volume boundaries). The resulting face-centered velocity field $\boldsymbol{u}_{n+1}^{f}$ is exactly non-divergent by construction. The cell-centered velocity field at time $n+1$ is obtained by applying a cell-centered pressure correction,

$$
\boldsymbol{u}_{n+1}^{c}=\boldsymbol{u}_{\star}^{c}-\left|\frac{\Delta t}{\rho\left(c_{n+\frac{1}{2}}^{f}\right)} \nabla^{f} p_{n+\frac{1}{2}}\right|^{c},
$$


where the $|.|^{c}$ operator denotes averaging over all the faces delimiting the control volume. The resulting cell-centered velocity field $\boldsymbol{u}_{n+1}^{c}$ is approximately divergence-free.

(3) The volume fraction is advected using an operator-split algorithm with the velocity field obtained above[37-40]. The volume flux needed for advection is achieved by performing a geometrical flux computation. A Mixed-Youngs-Centred $(M Y C)$ method for interface normal computation of Aulisa et al. [41] on a $3 \times 3 \times 3$ generalized for the octree spatial discretization has been employed for interface reconstruction.

(4) The surface tension force $\left(\sigma \kappa \delta_{s} \boldsymbol{n}\right)_{n+\frac{1}{2}}$ is calculated using a balanced-force surface-tension calculation [42]. A second-order accurate estimate of the curvature is obtained using the Height-Function technique [29]. In regions where the radius of curvature is smaller than five cells, a paraboloid fitting technique [29] is used. Details of the implementation are available elsewhere[28, 29, 34].

(5) An efficient mesh refinement and adaptation, usually costing less than $1 \%$ of computation time, is performed every time step using several criteria, for e.g., vorticity, gradient of a field variable or the curvature of the interface [28].

\section{B. Lagrangian particle advection}

Lagrangian particle tracking is performed using the updated velocity field. The fluid forces which are a function of the relative velocity between the particle and the fluid are computed. The fluid velocity is obtained at the particle position using a bilinear interpolation. The acceleration (Eq. 8) so obtained is integrated to compute the velocity of the particle and subsequently the updated particle position (Eq. 7). Equations (7) and (8) are discretized in time using the first-order explicit forward-Euler scheme.

\section{Two-way coupling force computation}

The two-way coupling force is a momentum source term in the Navier-Stokes equation (Eq. 14). To achieve numerical convergence it is smoothed using a Gaussian distribution with standard deviation $\sigma_{p}$. The standard deviation for the distribution of force is taken to be the maximum of the radius of the particle and the size of the computational cell containing its center. The particle size is in general smaller than the cell-size except in special situations when particles re-enter a highly refined region. The smoothed force is given by,

$$
\tilde{\boldsymbol{\Phi}}_{p}=\boldsymbol{\Phi}_{p} e^{-\left|\boldsymbol{x}-\boldsymbol{x}_{p}\right|^{2} / \sigma_{p}^{2}} /\left(\sqrt{2 \pi} \sigma_{p}\right)^{D},
$$


where $\tilde{\boldsymbol{\Phi}}_{p}$ is the smoothed force and $D$ is the dimension of the problem (2 in 2D).

The smoothed force is distributed only in a surrounding stencil of approximately $3 \sigma_{p}$ to reduce the computational cost. On a quad/octree grid we achieve the above criterion efficiently by moving to a coarser level till one of the cell-faces is at least $3 \sigma_{p}$ distance from the particle thence the closest neighbors in 2 (3 in 3-dimensional) directions are identified and the children of these are traversed up to the finest level where the source term $\Phi_{p}$ is defined. Figure 1 shows the parent cell satisfying the criterion layed above. The arrows mark the neighboring cells which should also be traversed for the smoothed force computation.

\section{Collisions}

Collisions between particles are predicted by using a spatial hashing algorithm in which a pointer to the cell is used as a key to the list of particles contained in it [43]. After each time-step, a hash-table is updated with the list of particles contained in a computational cell, the key to the elements of the hash-table being the pointer to the computational cell. For collision, particles residing in the neighboring cells are considered. Once the minimum collision time is established among all the possible collisions in the computational domain, as described below, the particles are advected for the minimum collision time between any two particles in the whole domain. The time-step of the NavierStokes solver is divided into several small advection time-steps based on the collisions occurring in the domain. The collision criterion described below is checked for all the possible pairs of particles in the adjacent cells. Collision occurs if $d t_{\text {coll }}$ defined below is finite,

$$
d t_{\text {coll }}=\left\{\begin{array}{l}
\infty \quad \text { if } \quad \boldsymbol{r}_{i j} \cdot \boldsymbol{v}_{i j}>0 \\
\infty \quad \text { if } d<0 \\
-\frac{\left(\boldsymbol{r}_{i j} \cdot \boldsymbol{v}_{i j}+\sqrt{d}\right)}{\boldsymbol{v}_{i j} \cdot \boldsymbol{v}_{i j}} \text { otherwise }
\end{array}\right.
$$

where $\boldsymbol{r}_{i j}=\boldsymbol{r}_{j}-\boldsymbol{r}_{i}$ and,

$$
d=\left(\boldsymbol{r}_{i j} \cdot \boldsymbol{v}_{i j}\right)^{2}-\left(\boldsymbol{r}_{i j} \cdot \boldsymbol{r}_{i j}-s^{2}\right)\left(\boldsymbol{v}_{i j} \cdot \boldsymbol{v}_{i j}\right) .
$$

Here, $s$ is the sum of the radii of the two particles, $\boldsymbol{r}_{i j}$ and $\boldsymbol{v}_{i j}$ are the relative position and velocities of the two particles for which collision is tested.

A hard-sphere elastic collision involves exchange of momentum conserving the momentum and the energy of the system of two particles. The time of interaction between two particles is considered infinitesimal with an impulsive force 
acting on the colliding particles. However, a collision between two droplets or bubbles is different from a hard-sphere collision with the outcome of the collision being a function of the kinetic energy, surface energy and the impact parameter of the droplets/bubbles among other factors. A priori prediction of the outcome of a collision can be made if the drop Weber number, the impact parameter (which defines the geometrical orientation of the collision for a pair of droplets) and the droplet-size ratio, are known [44, 45]. A pair of colliding droplets may coalesce, bounce (inelastically) or cross through each other deforming grossly and possibly breaking up into several smaller droplets [46]. A simple semi-empirical criterion can be employed to decide the post-collision scenario. Particle Stokes number, Weber number and the collision angles form the dominating collision-type deciding factors.

\section{E. Conversion of small droplets formed during atomization into particles}

A small Volume-of-Fluid resolved droplet, formed during the numerical simulations of the primary atomization, leads to expensive computational effort with little gain. In the present study, we propose to model small droplets as Lagrangian particles. The center of the particle is located at the centroid of the droplet and the average momentum is computed to define the velocity of the particle. The void fraction field is re-assigned to remove the droplet from the computational domain.

Similarly, a point particle formed from a droplet can be transformed back into a VOF-resolved droplet based on its proximity with the VOF interface or a pre-specified region. The two-way coupling force is replaced by the velocity impulse introduced in the computational domain upon the transformation of the particle into a VOF-resolved droplet. Subsequently, the refined computational grid containing the droplet is assigned a uniform velocity field corresponding to the momentum of the particle. The above approximation can be improved by choosing an approximate analytical solution for the flow field inside the droplet.

\section{Numerical Validation}

\section{A. Lagrangian particle tracking in a vortex}

For validation of the implementation of the Lagrangian particle-tracking algorithm, the motion of a particle computed using Gerris is compared with the results obtained from the Runge-Kutta integration using a prescribed shear- 
flow field. Only one-way coupling is used here. The fluid velocity field in the domain is assigned as,

$$
\begin{aligned}
& u=-C_{s} \sin (\pi y) \cos (\pi x), \\
& v=C_{s} \sin (\pi x) \cos (\pi y),
\end{aligned}
$$

The drag coefficient used is a function of the local particle Reynolds number $R e_{p}[16]$,

$$
C_{d}=\left\{\begin{array}{lc}
16 \frac{1+0.15 \sqrt{R e_{p}}}{R e_{p}} & \text { if } R e_{p}<50, \\
48 \frac{R e_{p}^{1 / 2}-2.21}{R e_{p}^{3 / 2}} & \text { otherwise. }
\end{array}\right.
$$

The lift and added-mass force coefficients are chosen to be one half. Table 1 shows the convergence of the error with refinement. The definition of the $L_{2}$ error norm used for comparison is,

$$
L_{2 \boldsymbol{q}}=\sqrt{\left(\frac{q x_{e}-q_{p}}{q x_{e}}\right)^{2}+\left(\frac{q y_{e}-q y_{p}}{q y_{e}}\right)^{2}},
$$

where $\boldsymbol{q}$ is the vector with $q x$ and $q y$ as components in $x$ and $y$ directions, respectively. The reference value is denoted by the subscript $e$ whereas subscript $p$ denotes the computed value for the particle.

The error in position and velocity of the particle has a first-order dependence on the grid resolution as we employ a first-order interpolation algorithm to obtain the fluid velocity to compute the fluid forces. Error shown in the table depicts the error in the velocity. Figure 2 shows the particle trajectory for different grid resolutions.

The cross in the figure marks the center of the anti-clockwise vortex. The solution obtained from the Runge-Kutta scheme is overlapping the computational results obtained for a grid resolution of $2^{8} \times 2^{8}$.

\section{B. Particle entrapment in a Rankine vortex}

Bubbles are captured and transported by vortices. Here we illustrate the effect of a Rankine vortex on bubbles rising vertically under buoyancy forces [30] . A Rankine vortex has a core (defined by its radius $a$ ) of uniform vorticity, say $2 \Omega$, and the exterior is irrotational. Only the azimuthal flow velocity is non-zero given by,

$$
u_{\theta}=\left\{\begin{array}{l}
\Omega r \text { for } r \leq a, \\
\Omega a^{2} / r \text { for } r>a .
\end{array}\right.
$$


The two key parameters governing the entrapment of the bubbles [30] are: (a) $\Pi=\Omega^{2} a / g$ which estimates the relative strength of the inertial force driving the particles towards the core of the vortex to the gravitational force and (b) the trapping parameter $S r=a \Omega / v_{T}$. Here, $v_{T}$ is terminal rise velocity of the bubbles in a quiescent liquid.

Figures 3 and 4 show planar (2D) simulations for two situations (i) $S r=2.0$, $\Pi=10.0$ and (ii) $S r=0.33$ and $\Pi=0.5$. For the first case the bubbles are influenced and trapped by the vortex to a stable point inside the vortex. This stable point can be obtained analytically by setting velocity and acceleration to zero in the governing equations for the particle motion. For a smaller entrapment parameter $(S r)$ and attracting parameter $\Pi$, the bubble path lines pass through the vortex only slightly deforming close to the Rankine vortex core.

\section{Source of momentum, $\boldsymbol{\Phi}_{p}$}

Forces on the fluid due to particles are modeled as a source of momentum in the Navier-Stokes equation. The size of the particle being considerably smaller compared to the computational grid size, the source of momentum is a pointforce. As discussed in section III.C., we diffuse the force in a small region. Here, we discuss a test case to evaluate the numerical aspects of diffusing a point force in a small region.

A point-source of momentum in a fluid leads to a jet of fluid flowing away from the source [47]. The flow generated is axi-symmetric with the line of force as the axis-of-symmetry. A non-dimensional analysis yields a Reynoldsnumber-like parameter $F /\left(2 \pi \rho \nu^{2}\right)$ governing the flow. The analytical solution for the fluid velocity is obtained by assuming a stream function $r \nu f(\theta)$. Here $r$ and $\theta$ are the radial and the azimuthal spherical coordinates. The function $f(\theta)$ is obtained by solving for the flow velocities satisfying the mass and the momentum conservation equations,

$$
f(\theta)=2 \frac{1-\cos ^{2}(\theta)}{1+C-\cos (\theta)}
$$

where $C$ is a constant of integration [47]. The flow-field so obtained corresponds to the one generated by a point force at the origin. The relation between the magnitude of the force $F$ and $C$, is

$$
\frac{F}{2 \pi \rho \nu^{2}}=\frac{32}{3} \frac{1+C}{C(2+C)}+4(1+C)^{2} \log \left(\frac{C}{2+C}\right)+8 C,
$$

where $\rho$ is the density of the fluid and $\nu$ is the kinematic viscosity. 
Due to the point source of momentum, a jet is formed by the entrainment of the slow-moving fluid pushed rapidly away from the origin. The edge of the so formed jet can be defined conveniently by the position where the streamlines are at the minimum distance from the axis. The semi-angle $\theta_{0}$ marks the azimuthal coordinate of the edge of the jet, where $\theta_{0}=\cos ^{-1}(1 /(1+C))$.

Interestingly, there is no inherent length-scale of the problem and the velocityfield diverges at the point-of-source. In numerical simulations (axi-symmetric), the length-scale of the problem is introduced by the size of the computational domain and the grid-size. We diffuse the point force in a small region using a Gaussian distribution for numerical convergence. Figure 5 shows the streamlines for $F / 2 \pi \rho \nu^{2}=50$. The theoretical result is $\theta_{0}=25^{\circ}$ and we obtain $\theta_{0}=30.2^{\circ}$ with a force diffused using a standard deviation of $2^{-10}$ (octgridlevel 10) after 200 units of time. Figure 6 shows the comparison of the axial velocity field at $\theta=\pi / 2$. For a grid refinement of level-8 [29] the velocity varies as $r^{-1.46}$, for level-9 refinement as $r^{-1.14}$ and for level-10 refinement as $r^{-0.97}$. The variation in the immediate vicinity of the force is $r^{-1}$ which is in agreement with the analytical result. The flow field in the whole simulation domain adapts slowly and a good comparison between the numerical simulations and the theoretical result is obtained at large dimensionless times $(t=300)$. Figure 7 shows the streamlines for $F / 2 \pi \rho \nu^{2}=0.1$ for which a large $\theta_{0}(\sim \pi / 2)$ is obtained which is in agreement with the theoretical result [48].

To show the effect of the two-way coupling force in the presence of a large concentration of the particles we show here a simulation of the particles in a row rising under gravitational force in a viscous fluid. The series of particles is injected with a zero velocity at a high feed rate $(f=20$ particles per sec. per location). The two-way coupling leads to a momentum transfer to the surrounding initially quiescent liquid. The particles arriving in the agitated liquid respond with a mixing layer type instability as is visible in Figure 8 from the position of the particles. The non-dimensional parameter governing the flow is $\nu f /(l g)=1.05 \times 10^{-3}$ where $\nu$ is the viscosity of the fluid, $l=0.2 \mathrm{~m}$ is the width of the line of particles and $g=-10 \mathrm{~m} / \mathrm{s}^{2}$ is the gravitational constant. The particle-liquid density ratio is $10^{-3}$. The drag coefficient used is described by equation (27).

\section{Simulations of primary jet break-up}

A liquid jet atomizes into small droplets by a high-speed coaxial air-jet flowing over it. Thin liquid ligaments form at the liquid-gas interface due to the instability. Subsequently, the ligaments break into droplets. The prediction of the droplet size distribution in an atomization process is of immense importance in several industrial applications. For example, the distribution of fuel 
in combustion chambers is critical to fuel economy.

A numerical simulation of atomization of a liquid jet resolving the smallest droplets becomes progressively computationally expensive with the formation of droplets. As an approximation, the droplets can be removed from the computational domain assuming the negligible effect on the atomization process [34]. However, in the process, the subsequent motion of the droplets in the simulation chamber is not accounted for. Thus, reliable measurements of the PDF of the droplet sizes are not possible. In the present study, we model the droplets as Lagrangian particles moving under the influence of fluid forces.

In the present section, we discuss an atomization simulation, performed on a parallel machine using eight nodes, with small droplets transformed into Lagrangian particles.

A planar simulation of a liquid jet destabilized by a high-speed co-flowing gas has been performed. A thin tapered separator plate (Figure 9) separates the liquid and gas flow near the inlet as in the experiments of Cartellier et al. [49]. The taper angle (= half angle of the wedge) used in the present simulation is $3.5^{\circ}$ and the thickness of the separator plate at the inlet is $150 \mu \mathrm{m}$. The flow parameters of the liquid and gas used are given in Table 2. The nondimensional parameters governing the process are, liquid-gas momentum ratio $M=\rho_{g} U_{g}^{2} / \rho_{l} U_{l}^{2}=16$, gas and liquid Reynolds numbers $\left(R e_{g}=\rho_{g} U_{g} \delta_{g} / \mu_{g}=2060\right.$ and $\left.R e_{l}=\rho_{l} U_{l} \delta_{l} / \mu_{l}=5000\right)$ and Weber number $W e_{g}=\rho_{g} U_{g}^{2} \delta_{g} / \sigma=10.2$. The thickness of the boundary layer in the gas, $\delta_{g}=6.05 R e_{g}^{-1 / 2} H_{g}$, at the nozzle inlet is a function of $R e_{g}$. It is prescribed at the nozzle inlet using an experimental correlation [50]. Here, $H_{g}$ is the thickness of the gas jet. A similar correlation has been used at the liquid inlet to obtain $\delta_{l}$. The smallest grid size in the simulation is $H_{g} / 2048$ for resolving the interface. The interface and the separator plates are resolved with the finest grid cells. The criterion for adaptive refinement based on local vorticity is different in different regions.

The mechanisms leading to the atomization of a jet are a topic of current active research. One mechanism involves a predominant role of the gas phase. The study of the stability of a two-phase mixing layer shows that the interface becomes unstable when the relative velocity of gas and liquid is sufficiently high compared to surface tension. Crests of the instability at the liquid-gas interface gain momentum from the gas jet leading to the roll-up of the interface, and the formation of thin ligaments. Further, ligaments break into droplets. We model the droplets so formed during atomization by transforming them into particles to be tracked with the Lagrangian particle model described in Sec. III.. Such a transformation is performed for any droplets in the computational domain that occupy less than 25 computational cells. The local computational grid upon transformation is coarsened. 
Figure 10 shows a snapshot of an atomization simulation with parameters in Table 2 and non-dimensional parameters mentioned before. The dots in the figure represent droplets which have been modeled as Lagrangian particles. Droplets once formed are entrained into the fast moving air-stream and are scattered in a wider region. The particles formed from droplets are resolved again by VOF when they approach a VOF-resolved interface. The sequence in Fig. 11 represents two such scenarios encountered during the simulation. The droplet upon hitting the interface causes a splash and may initiate a fragmentation of the bigger lump of liquid into which it collided. In the upperhalf of the figure a collision between a particle and a smaller lump of liquid causes breakup of the liquid structure into smaller fragements. Therefore, such interactions can modify the droplet size distribution.

Figure 12 shows the particle trajectories of the droplets formed near the inlet. The line-color represents the diameter of the particles marked in the legend. The particle lines maintain a near-constant angle with the mean jet flow. A few particles have a shorter life span before they collapse back into the liquid jet.

Figures 13(a) and 13(b) show the probability density distributions (PDF) of the droplets in two different regions of the computational domain (a) close to the nozzle inlet and (b) far from it (marked in Fig. 10). Figure 13(a) shows a rather sharp peak for a diameter $(10-20 \mu \mathrm{m})$ and another smoother peak of the distribution for a diameter $(40-50 \mu \mathrm{m})$. In contrast, Fig. 13(b) shows a clear peak of the distribution at $\sim 120 \mu \mathrm{m}$ suggesting formation of larger droplets away from the nozzle. The above PDFs, thus, delineate the two mechanisms of formation of droplets, namely, by primary atomization of the jet and another by break-up of bigger fragments of liquid emerging downstream.

\section{Conclusions}

In the present study we performed multiscale numerical simulations by combining a Volume-of-Fluid (VOF) algorithm with a Lagrangian particle tracking algorithm. The two-way coupling between Lagrangian particles and the fluid incorporated the loss or gain of momentum in the fluid because of the presence of particles. A spatial algorithm has been implemented for efficient and speedy prediction of possible collisions between particles. The algorithm has been validated with a set of test cases.

Employing the algorithm for the numerical simulations of atomization of a liquid jet by a high-speed coaxial gas flow, we transformed small droplets needing high grid resolution into Lagrangian particles. The atomization simulation presented in the current study has been performed for a high liquid-gas 
density ratio of 100 and a high momentum ratio of 16 . The process reduces the computational cost considerably, enabling us to simulate a swarm of droplets spreading outward from the liquid jet. The trajectories of the droplets formed show a near constant angle with the mean jet flow. A PDF of the droplet sizes in two different zones of the computational domain (near the nozzle inlet and far from it) delineates the two mechanisms of droplet formation in the atomization process.

\section{Acknowledgments}

\section{References}

[1] K. Kusano, A. Kawano, H. Hasegawa, Macro-micro interlocked simulations for multiscale phenomena, in: International Conference on Computational Science, 2007.

[2] V. I. Kolobov, R. R. Arslanbekon, A. V. Vasenkov, Coupling atomistic and continuum models for multi-scale simulations, in: International Conference on Computational Science, 2007.

[3] J. Fish, W. Chen, Discrete-to-continuum bridging based on multigrid principles, Comp. Meth. Appl. Mech. Engng. 193 (2004) 1693 - 1711.

[4] W. E. Engquist, B. Engquist, The heterogeneous multi-scale methods, Comm. Math. Sci. 1 (2002) $87-132$.

[5] C. Helzel, F. Otto, Multiscale simulations for suspensions of rod-like molecules, J. Comput. Phys. 216 (2006) $52-75$.

[6] T. Y. Hou, X. Wu, A multiscale finite element method for elliptical problems in composite materials and porous media, J. Comput. Phys. 134 (1997) $169-189$.

[7] T. J. R. Hughes, Multiscale phenomena; Green's functions, the Dirichletto-Neumann formulation, subgrid scale models, bubbles and the origins of the stabilized methods, Comm. Meth. Appl. Mech. Engrg. 127 (1995) $387-401$.

[8] G. Son, N. Ramanujapu, V. K. Dhir, Numerical simulation of bubble merger process on a single nucleate site during pool nucleate boiling, J. Heat Transfer 124 (2002) 51.

[9] M. Chiesa, V. Mathisen, J. Melheim, B. Halvorsen, Numerical simulation of particulate flow by the Eulerian-Lagrangian and the Eulerian-Eulerian approach with application to fluidised bed, Computer and Chemical Engineering 29 (2005) 291.

[10] S. Elghobashi, G. C. Truesdell, Direct simulation of particle dispersion in a decaying isotropic turbulence, J. Fluid Mech. 242 (1992) 655-700.

[11] A. Ferrante, S. Elghobashi, On the effects of microbubbles on the TaylorGreen vortex flow, J. Fluid Mech. 572 (2007) 145-177. 
[12] M. van Sint Annaland, N. G. Deen, J. A. M. Kuipers, Numerical simulation of gas-liquid-solid flows using a combined front tracking and discrete particle method, Chemical Engineering Science 60 (2005) 6188-6198.

[13] T. Tanaka, J. K. Eaton, Classification of turbulence modification by dispersed sphers using a novel dimensionless number, Phys. Rev. Lett. 101 (2008) 114502.

[14] D. Lohse, Particles go with the flow, Physics 18 (2008) 1-4.

[15] M. R. Maxey, E. J. Chang, L. P. Wang, Simulations of interactions between microbubbles and turbulent flows, Appl. Mech Rev. 47 (1994) 7074.

[16] E. Climent, J. Magnaudet, Dynamics of a two-dimensional upflowing mixing layer seeded with bubbles: Bubble dispersion and effect of two-way coupling, Phys. Fluids 18 (2006) 103304.

[17] J. Brackbill, D. B. Kothe, C. Zemach, A continuum method for modeling surface tension, J. Comput. Phys. 100 (1992) 335-354.

[18] S. O. Unverdi, G. Tryggvason, A front-tracking method for viscous, incompressible, multi-fluid flows, J. Comput. Phys. 100 (1992) 25-37.

[19] M. Sussman, P. Smereka, S. Osher, A level set approach for computing solutions to incompressible two-phase flow, J. Comput. Phys. 114 (1994) 146-159.

[20] S. Zaleski, J. Li, R. Scardovelli, G. Zanetti, Flows with interfaces: dealing with surface tension and reconnection, in: Proceedings of the IMACSCOST Conference on Computational Fluid Dynamics, Lausanne Sept. 13-15, 1995,Notes on Numerical Fluid Mechanics, Vieweg, 53, 1995.

[21] S. Popinet, S. Zaleski, A front tracking algorithm for the accurate representation of surface tension., Int. J. Numer. Meth. Fluids 30 (1999) 775-793.

[22] J. E. Pilliod Jr, E. G. Puckett, Second-order accurate volume-of-fluid algorithms for tracking material interfaces, Tech. rep., Lawrence Berkeley National Laboratory, no. LBNL-40744, also submitted to J. Comput. Phys (1997).

[23] Y. Renardy, M. Renardy, PROST - A parabolic reconstruction of surface tension for the volume-of-fluid method, J. Comput. Phys. 183 (2002) 400421.

[24] F. K. Keller, J. Li, A. Vallet, D. Vandromme, S. Zaleski, Direct numerical simulation of interface breakup and atomization, in: Proc. Sixth Int. Conf. on Liquid Atomization and Spray Systems, Rouen, 1994, pp. 56-62.

[25] W. Tauber, G. Tryggvason, Direct numerical simulation of primary breakup, Comput. Fluid Dynamics J. 9 (2000) 594.

[26] D. Kim, M. Herrmann, P. Moin, The breakup of a round liquid jet by a coaxial flow of gas using the refined level set grid method, in: American Physical Society, 59th Annual Meeting of the APS Division of Fluid Dynamics, November 19-21, 2006, p. FB.010.

[27] S. Zaleski, T. Boeck, Direct numerical simulation of high speed jet atomization, in: International Conference on Liquid and Spray Systems 
(ICLASS) 2003, Sorrento, Italy, 2003.

[28] S. Popinet, Gerris: a tree-based adaptive solver for the incompressible Euler equations in complex geometries, J. Comput. Phys. 190 (2003) 572600.

[29] S. Popinet, An accurate adaptive solver for surface-tension-driven interfacial flows, Journal of Computational Physics 228 (2009) 5838-5868.

[30] J. Magnaudet, I. Eames, The motion of high-Reynolds-number bubbles in inhomogeneous flows, Ann. Rev. Fluid Mech. 32 (2000) 659-708.

[31] D. Legendre, J. Magnaudet, The lift force on a spherical bubble in a viscous linear shear flow, J. Fluid Mech. 368 (1998) 81-216.

[32] J. Magnaudet, M. Rivero, J. Fabre, Accelerated flows around a rigid sphere or a spherical bubble. part i: Steady straining flow, Journal of Fluid Mechanics 284 (1995) 97-136.

[33] T. R. Auton, The lift force on a spherical body in a rotational flow, J. Fluid Mech. 183 (1987) 199-218.

[34] D. Fuster, A. Bague, T. Boeck, L. Moyne, A. Leboissetier, S. Popinet, P. Ray, R. Scardovelli, S. Zaleski, Simulation of primary atomization with an octree adaptative mesh refinement and vof method, Int. J. Multiphase Flow 35 (2009) 550-565.

[35] J. Bell, P. Colella, H. Glaz, A second-order projection method for the incompressible Navier-Stokes equations, J. Comput. Phys. 85 (1989) 257283.

[36] A. Almgren, J. Bell, W. Crutchfield, Approximate projection methods: part I. inviscid analysis, SIAM Journal on Scientific Computing 22 (2000) 1139-1159.

[37] R. DeBar, Fundamentals of the KRAKEN code, Tech. rep., California Univ., Livermore (USA). Lawrence Livermore Lab. (1974).

[38] W. Noh, P. Woodward, SLIC (simple line interface calculation), in: A. van de Vooren, P. Zandbergen (Eds.), Proceedings, Fifth International Conference on Fluid Dynamics, Vol. 59 of Lecture Notes in Physics, Springer, Berlin, 1976, pp. 330-340.

[39] J. Li, Calcul d'interface affine par morceaux (piecewise linear interface calculation), C. R. Acad. Sci. Paris, série IIb, (Paris) 320 (1995) 391-396.

[40] E. G. Puckett, A. S. Almgren, J. B. Bell, D. L. Marcus, W. J. Rider, A high-order projection method for tracking fluid interfaces in variable density incompressible flows, J. Comput. Phys. 130 (1997) 269-282.

[41] E. Aulisa, S. Manservisi, R. Scardovelli, S. Zaleski, Interface reconstruction with least-squares fit and split advection in three-dimensional cartesian geometry, J. Comput. Phys. 225 (2007) 2301-2319.

[42] M. M. Francois, S. J. Cummins, E. D. Dendy, D. B. Kothe, J. M. Sicilian, M. W. Williams, A balanced-force algorithm for continuous and sharp interfacial surface tension models within a volume tracking framework, J. Comput. Phys. 213 (2006) 141-173.

[43] E. Perkins, Spatial reasoning for general n-body physics - discrete element algorithms, MS Thesis, Massachusetts Institute of Technology, 
Cambridge, MA, USA. (1999).

[44] A. Menchaca-Rocha, A. Cuevas, M. Chapa, M. Silva, Rotating-liquiddrop model limit tested on macroscopic drops, Phys. Rev. E 47 (1993) 1433-1436.

[45] A. Mannannur, R. D. Reitz, A new predictive model for fragmenting and non-fragmenting binary droplet collisions, Int. J. Multiphase Flow 33 (2007) 873-896.

[46] S. Blei, C. A. Ho, M. Sommerfeld, A stochastic droplet collision model with consideration of impact efficiency, in: ILASS-Europe'98, Zaragoza, Spain, 2002.

[47] G. K. Batchelor, An introduction to fluid dynamics, Cambridge Univ. Press, 1970.

[48] L. D. Landau, A new exact solution of Navier-Stokes equations, C.R. (Doklady) Acad. Sci. URSS 43 (1944) 286-288.

[49] M. N. Descamps, J.-P. Matas, A. Cartellier, Gas-liquid atomisation: gas phase characteristics by piv measurements and spatial evolution of the spray, in: 2nd Colloque INCA, Rouen, France, 2008.

[50] F. Ben Rayana, Contribution à l'étude des instabilités interfaciales liquide-gaz en atomisation assistée et taille de gouttes, Ph.D. thesis, Institut National Polytechnique de Grenoble (2007). 
Table 1

Convergence of the error in the position and the velocity of a particle in a vortex

\begin{tabular}{ccc}
\hline Grid & Error $L_{2 \boldsymbol{x}}($ in $\%)$ & Error $L_{2 \boldsymbol{u}}($ in $\%)$ \\
\hline $2^{4} \times 2^{4}$ & 3.36 & 5.11 \\
$2^{5} \times 2^{5}$ & 1.36 & 2.16 \\
$2^{6} \times 2^{6}$ & 0.89 & 0.15 \\
$2^{8} \times 2^{8}$ & 0.09 & 0.08 \\
\hline
\end{tabular}


Table 2

Properties of the liquid and the coaxially flowing gas jet.

\begin{tabular}{cccc}
$U_{g} / U_{l}$ & $\rho_{l} / \rho_{g}$ & $\mu_{l} / \mu_{g}$ & $\delta_{g} / e$ \\
\hline 40 & 100 & 588 & 1.17
\end{tabular}




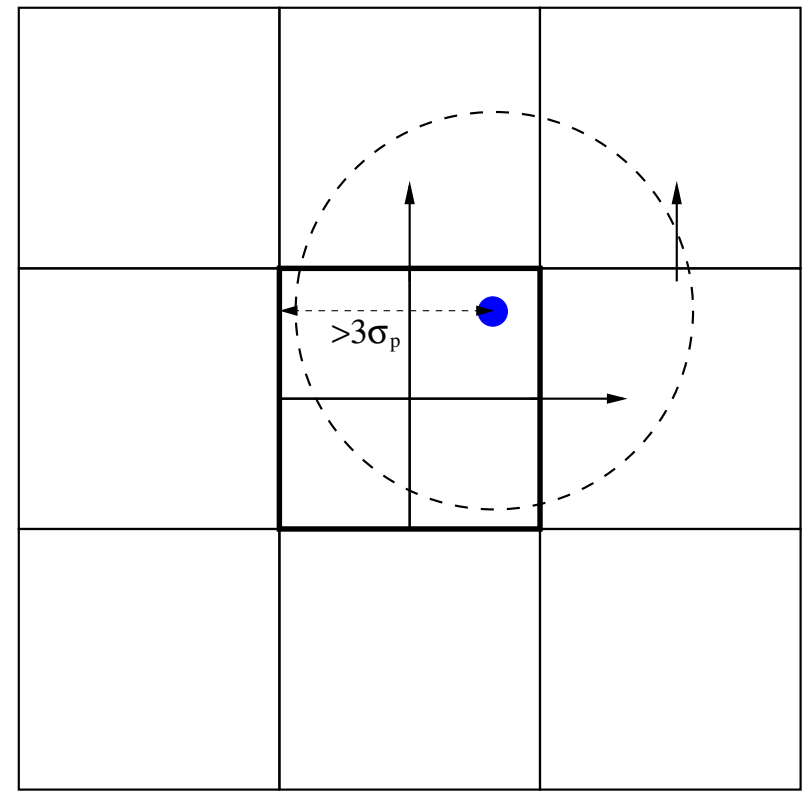

Fig. 1. Parent cells of which the leaf cells are traversed for the distribution of $\tilde{\boldsymbol{\Phi}}_{p}$. The parent cell marked with thicker border is identified by moving from finer to coarser grid level satisfying the criterion that one of the cell-face is at a distance greater than $3 \sigma_{p}$. The arrows mark the neighboring cells which are also traversed for smoothed force computation. 


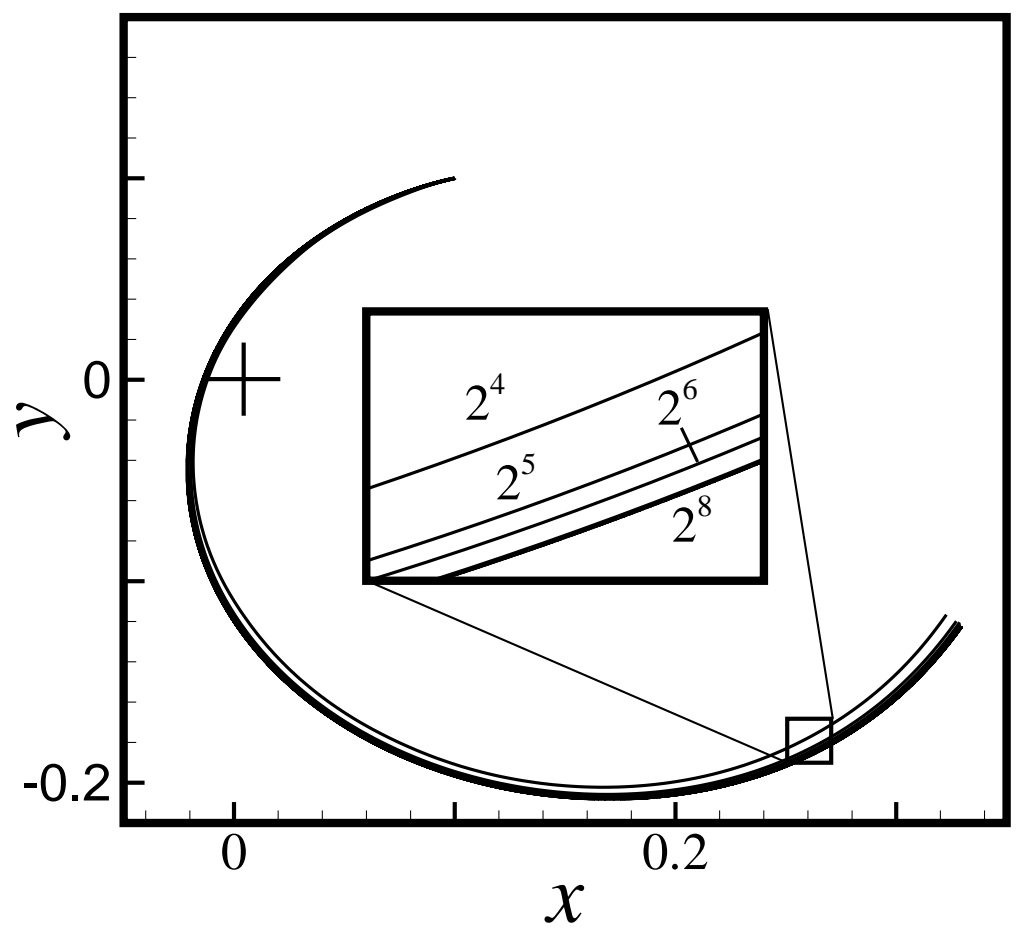

Fig. 2. Particle tracking in a vortex flow 


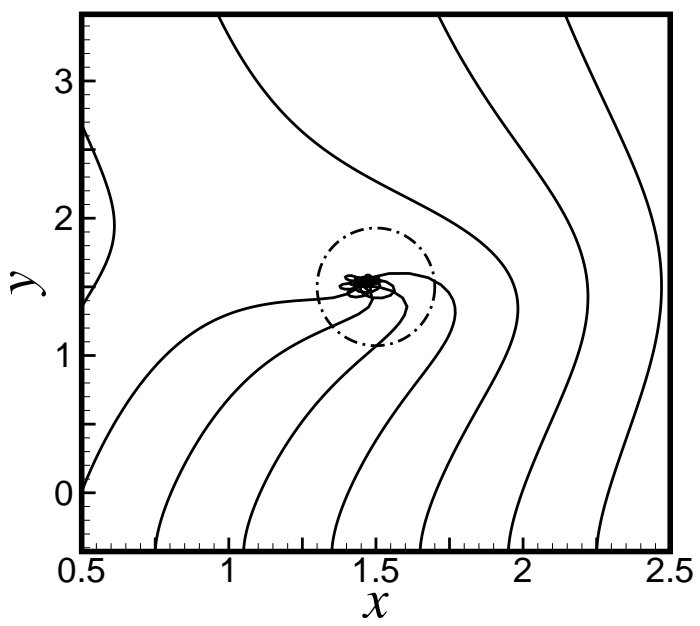

Fig. 3. Particle entrapment in a Rankine vortex with $S r=2$ and $\Pi=10$. 


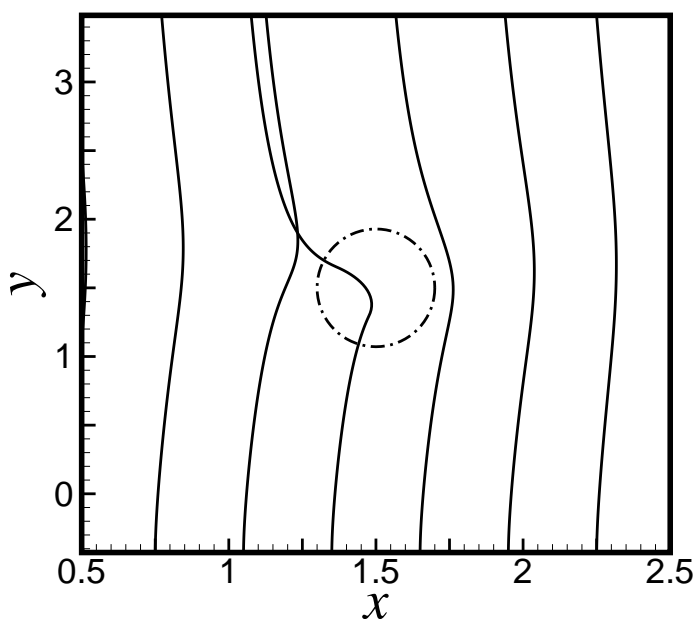

Fig. 4. Particle trajectories through a Rankine vortex with $S r=0.33$ and $\Pi=0.5$. 


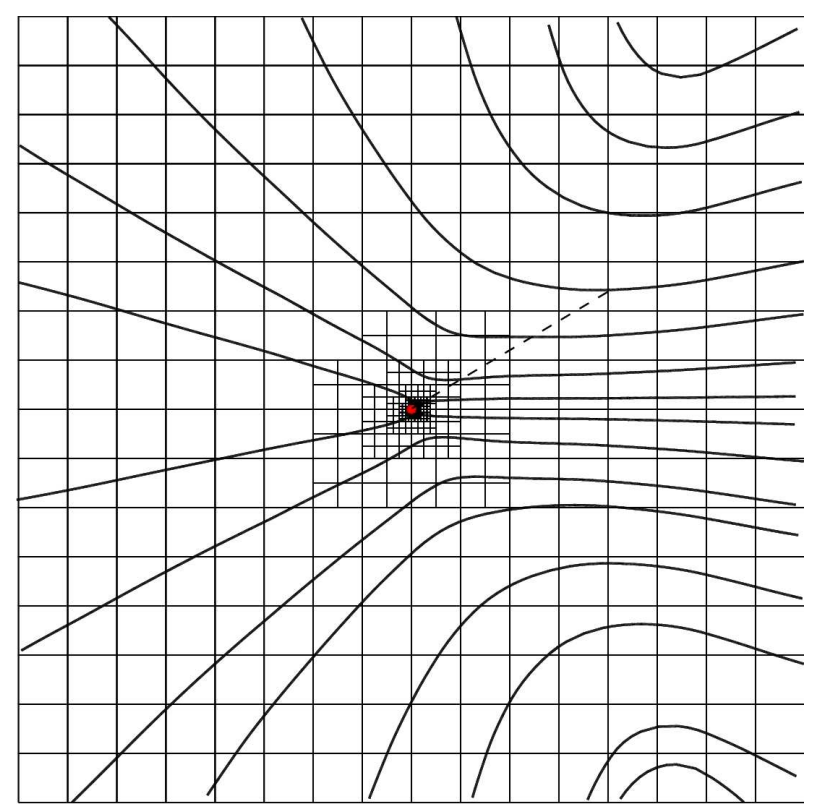

Fig. 5. Streamlines for a point force corresponding to $F /\left(2 \pi \rho \nu^{2}\right) \sim 50$ 


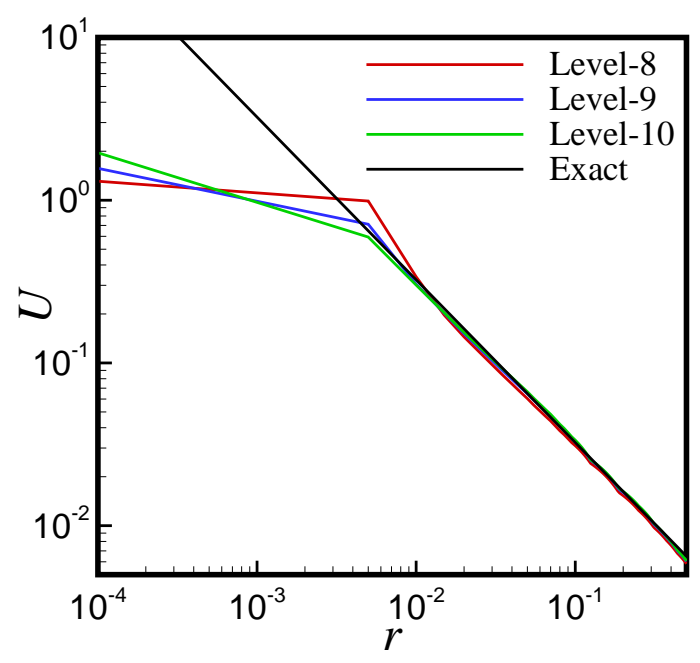

Fig. 6. Comparison of computed axial-velocity (at $\theta=\pi / 2$ ) for $F /\left(2 \pi \rho \nu^{2}\right) \sim 50$ for different grid-resolutions with the analytical solution of Landau[48]. 


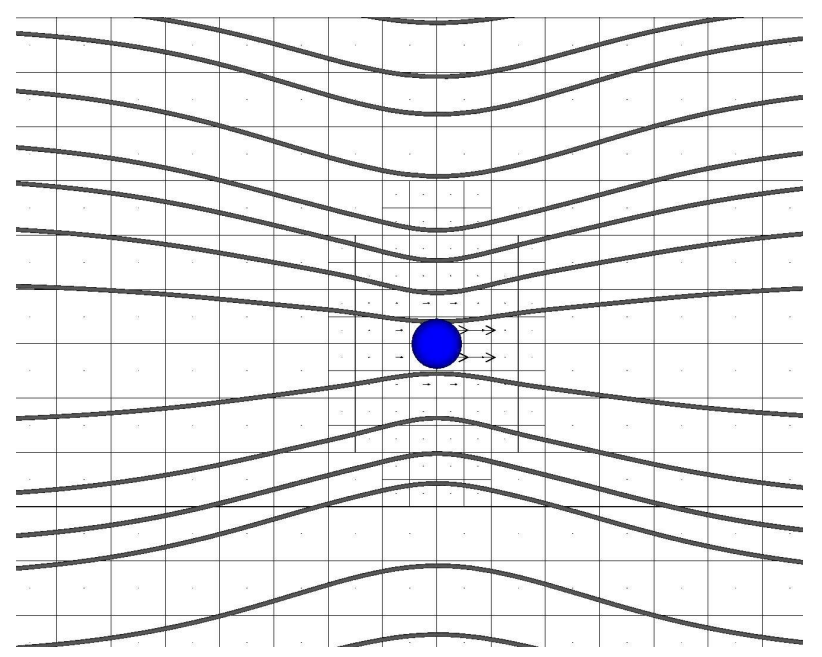

Fig. 7. Streamlines for a point force corresponding to $F /\left(2 \pi \rho \nu^{2}\right) \sim 0.1$ 


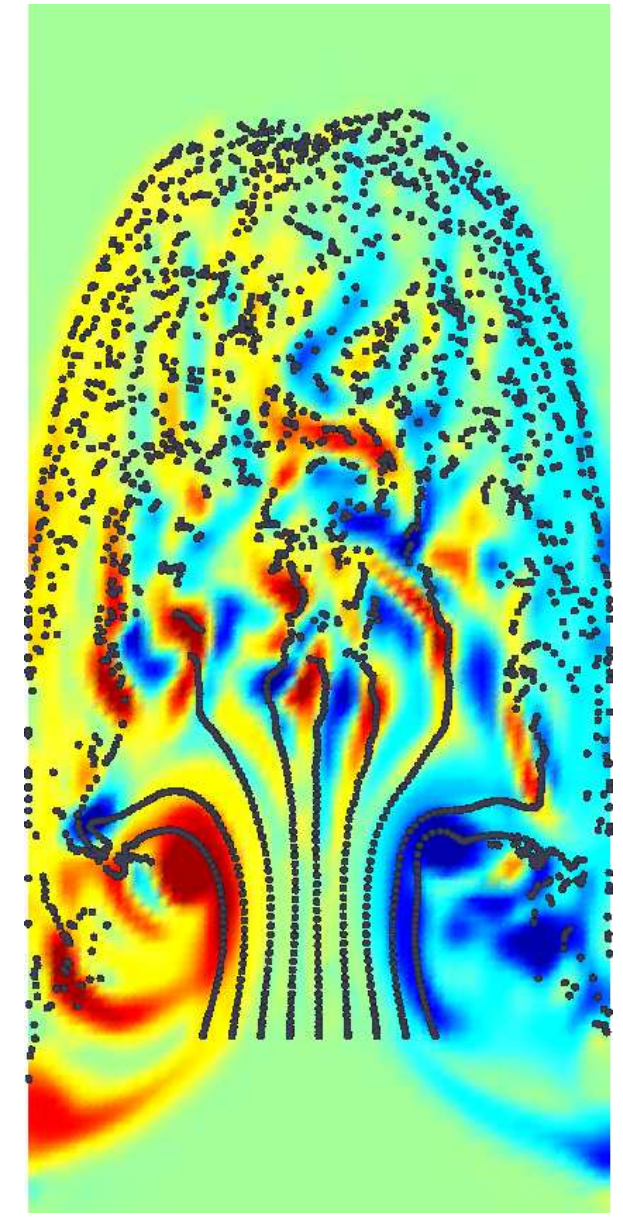

Fig. 8. A plume of particles (bubbles) in a viscous fluid 


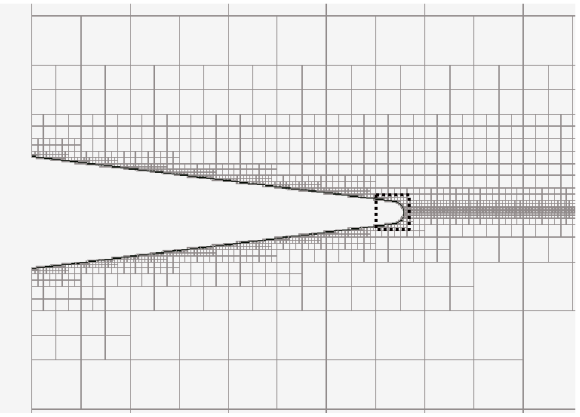

(a)

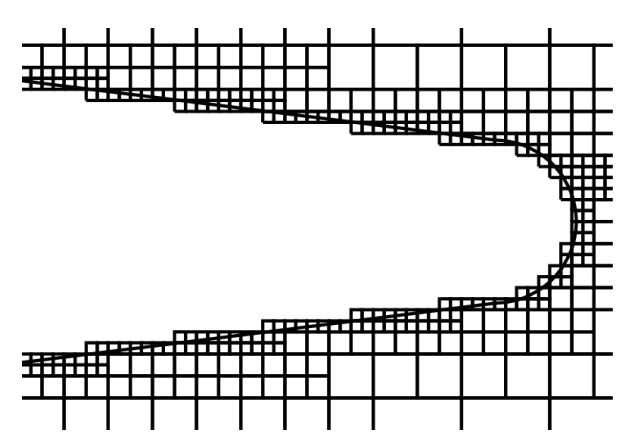

(b)

Fig. 9. (a) Shape of the nozzle separating the gas and liquid jets near the inlet. (b) Nozzle boundary cutting through the Eulerian grid cells. Cut cell method has been employed to impose the no-slip boundary condition accurately at the nozzle surface $[28]$. 


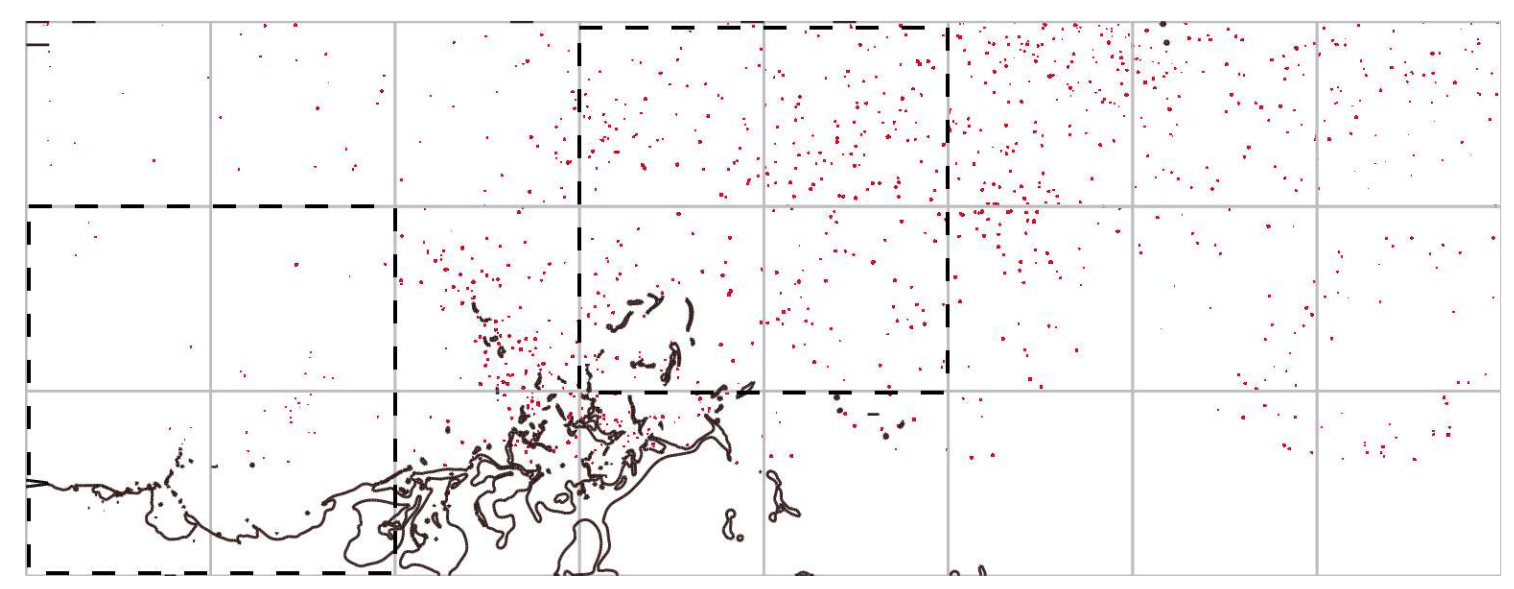

Fig. 10. Break-up of a liquid jet by a high-speed coaxially flowing gas-jet. A cloud of small droplets, modeled as Lagrangian particles (shown in red), formed during the atomization process are advected and spread by the high speed gas. The two dash boxes mark regions where we perform PDF of droplets. 


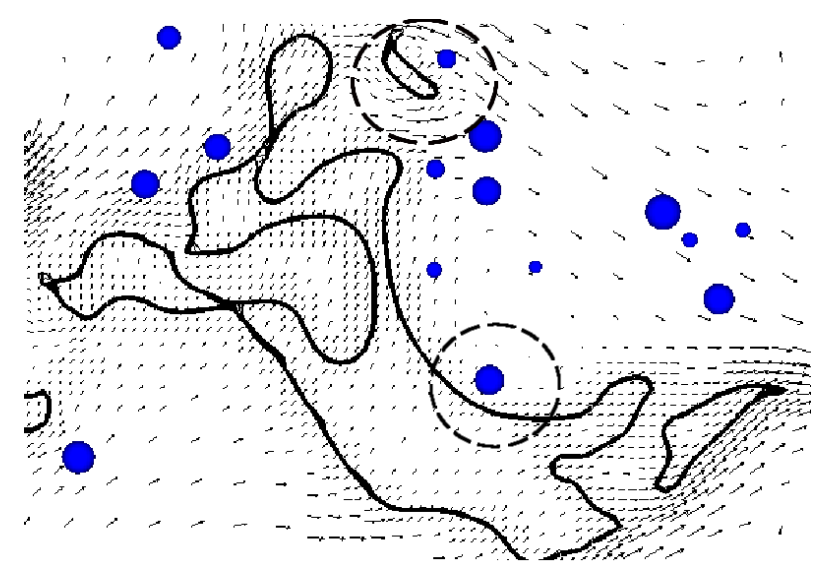

(a)

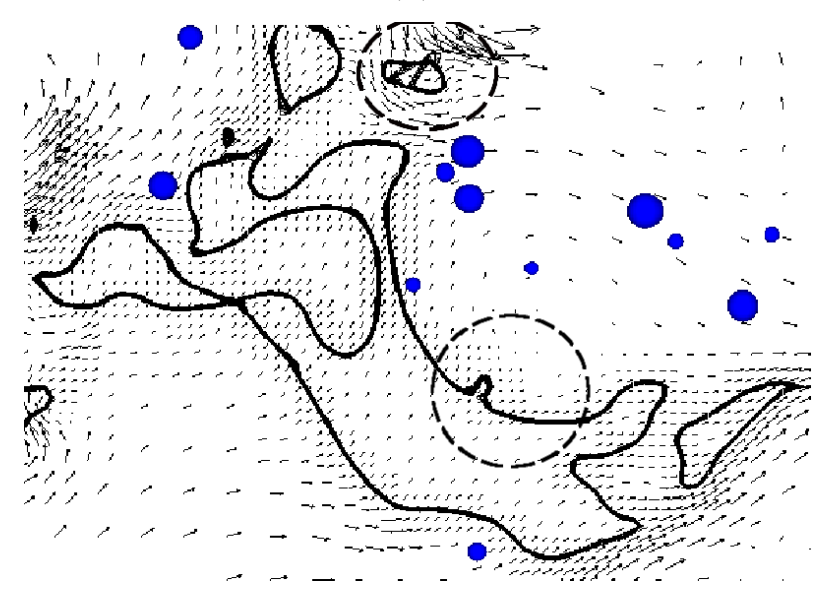

(b)

Fig. 11. (a) A close view of the distribution of the droplets transformed (b) An instant when a transformed droplet hits back a VOF resolved interface. 


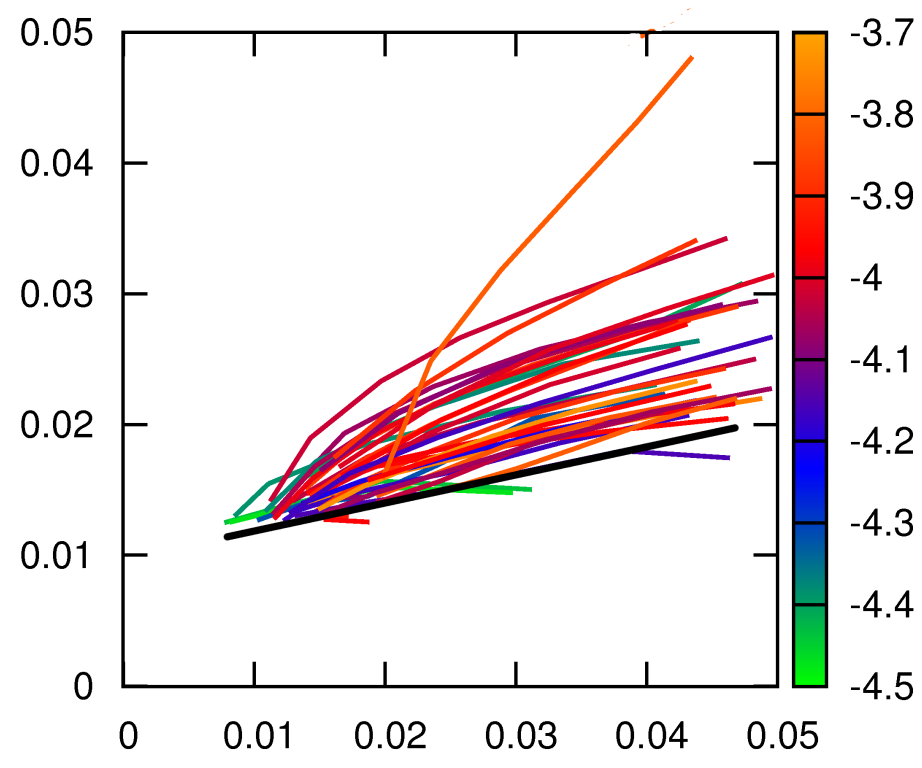

Fig. 12. Trajectories of the particles formed near the nozzle-inlet. The color of the trajectories correspond to logarithm (base 10) of the particle diameter with the adjoining color-index. The dark line shows the mean-trajectory $\left(\sim 18^{\circ}\right)$. 


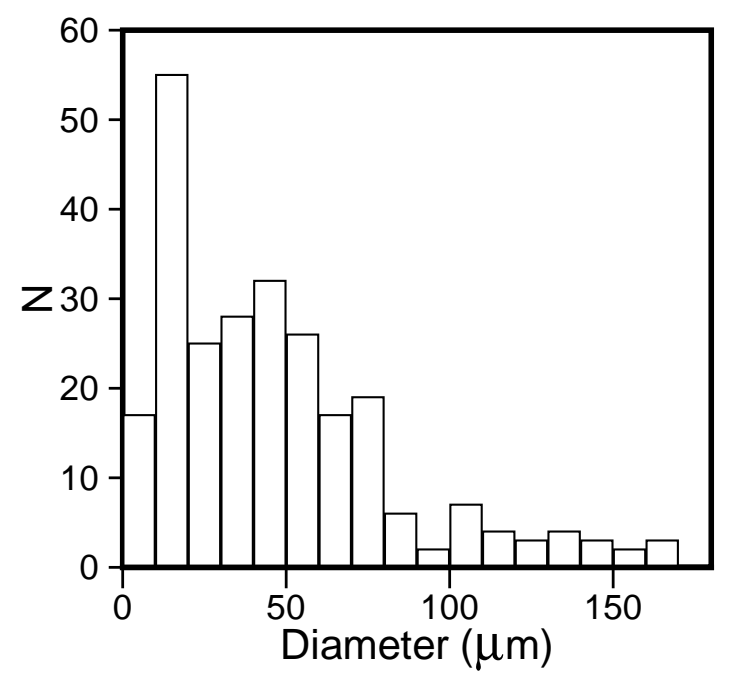

(a)

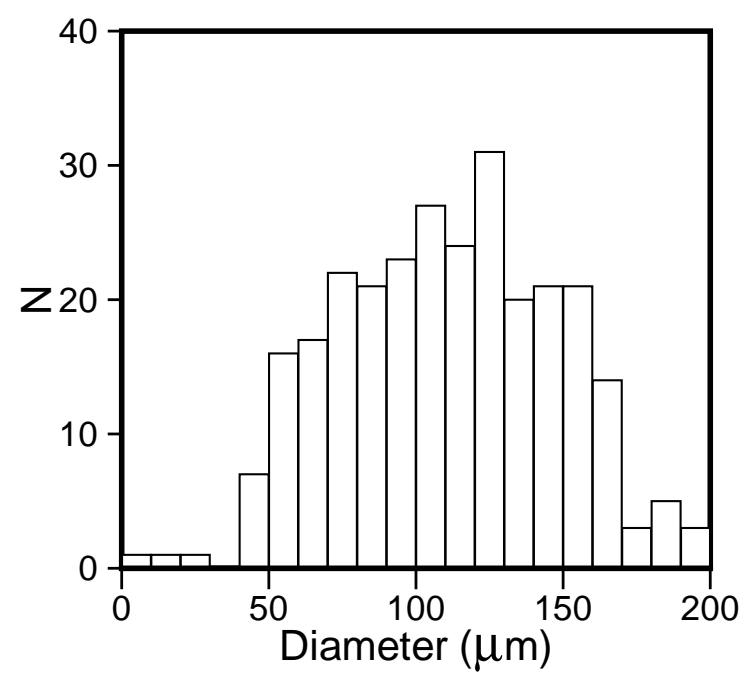

(b)

Fig. 13. Probability density distributions of the diameter of the droplets formed at a location (a) near the nozzle-inlet (b) further downstream as marked in the Figure 10. The abscissa of the plots show the diameter of the droplets in $\mu \mathrm{m}$. 